\title{
Advance in Drug Delivery for Ageing Skeletal Muscle
}

\author{
Yi $\mathrm{Li}^{1,2 \dagger}$, Ming Chen ${ }^{1,2 \dagger}$, Yanpeng Zhao ${ }^{1,2}$, Ming $\mathrm{Li}^{1,2}$, Yong $\mathrm{Qin}^{3}$, Shi Cheng ${ }^{3}$, \\ Yanyu Yang ${ }^{4 *}$, Pengbin Yin ${ }^{1,2 *}$, Licheng Zhang ${ }^{1,2^{*}}$ and Peifu Tang ${ }^{1,2}$ \\ ${ }^{1}$ Department of Orthopedics, General Hospital of Chinese PLA, Beijing, China, ${ }^{2}$ National Clinical Research Center for \\ Orthopedics, Sports Medicine \& Rehabilitation, Beijing, China, ${ }^{3}$ The Department of Orthopedic Surgery, Second Affiliated \\ Hospital of Harbin Medical University, Harbin, China, ${ }^{4}$ College of Materials Science and Engineering, Zhengzhou University, \\ Zhengzhou, China
}

\section{OPEN ACCESS}

Edited by:

Jianxun Ding,

Chinese Academy of Sciences, China

Reviewed by:

Hua Lu,

Peking University, China

Ruinan Yang,

Pharmaceutical Product

Development, United States

Xue Qu,

East China University of Science and

Technology, China

*Correspondence:

Yanyu Yang

yyyang@zzu.edu.cn

Pengbin Yin

yinpengbin@gmail.com

Licheng Zhang

zhanglcheng218@126.com

${ }^{t}$ These authors have contributed equally to this work

Specialty section:

This article was submitted to Translational Pharmacology, a section of the journal

Frontiers in Pharmacology

Received: 13 March 2020 Accepted: 23 June 2020 Published: 08 July 2020

Citation:

Li Y, Chen M, Zhao Y, Li M, Qin Y,

Cheng $S$, Yang $Y$, Yin $P$, Zhang $L$ and

Tang $P$ (2020) Advance in Drug

Delivery for Ageing Skeletal Muscle.

Front. Pharmacol. 11:1016.

doi: 10.3389/fphar.2020.01016
The age-related loss of skeletal muscle, sarcopenia, is characterized by progressive loss of muscle mass, reduction in muscle strength, and dysfunction of physical performance. It has become a global health problem leading to several adverse outcomes in the ageing population. Research on skeletal muscle loss prevention and treatment is developing quickly. However, the current clinical approaches to sarcopenia are limited. Recently, novel drug delivery systems offer new possibilities for treating aged muscle loss. Herein, we briefly recapitulate the potential therapeutic targets of aged skeletal muscle and provide a concise advance in the drug delivery systems, mainly focus on the use of nanocarriers. Furthermore, we elaborately discuss the prospect of aged skeletal muscle treatment by nanotechnology approaches.

Keywords: ageing skeletal muscle, drug delivery, extracellular vesicle, sarcopenia, skeletal muscle regeneration

\section{INTRODUCTION}

Skeletal muscles, as one of the largest organs in the body, are characterized by their mechanical activity required for posture, movement, and breathing (Fan et al., 2013; Pedersen, 2013). However, with the increasing age, skeletal muscle mass gradually declines and gradually loses function, leading to adverse outcomes in older people, such as high risk of falls and fractures, and damaged physical ability (Wilkinson et al., 2018; Cuesta-Triana et al., 2019). Current clinical approaches to age-related muscle loss are limited. At present, there are many studies on skeletal muscle regeneration which focus on muscle injuries, but research on strategies for age-related muscle loss is still relatively basic.

Conventionally, the research of skeletal muscle injuries mainly focuses on tissue engineering methods and cell therapies. These methods are suitable for the injury with obvious muscle defects (McKeon-Fischer et al., 2015). However, these methods are not suitable for age-related muscle loss, a kind of systemic skeletal muscle disease without local defect. In recent years, novel nano-carriers delivery systems offer new possibilities for treating systemic diseases. Even further, Rong, $S$ et al. proposed a prospect that extracellular vesicles (EVs), including exosomes, may also be ideal drug carriers which could regulate muscle regeneration and protein synthesis. EV-based delivery systems may be potential strategies of age-related muscle loss in the future (Rong et al., 2020).

Therefore, this review will discuss the potential therapeutic targets of ageing skeletal muscle and provide a concise advance in the drug delivery systems, mainly focus on the use of nano-carriers. 


\section{MOLECULAR MECHANSIM OF AGEING SKELETAL MUSCLE REGENERATION}

\section{The Process of Skeletal Muscle Regeneration}

Skeletal muscle regeneration includes four consecutive phases: the degeneration phase, the inflammatory phase, the phase of satellite cell differentiation, and the phase of maturation and remodeling (Barberi et al., 2013; Yin et al., 2013; Schmidt et al., 2019)

(Figure 1). After injury, myofibers are rapid necrosis (phase of

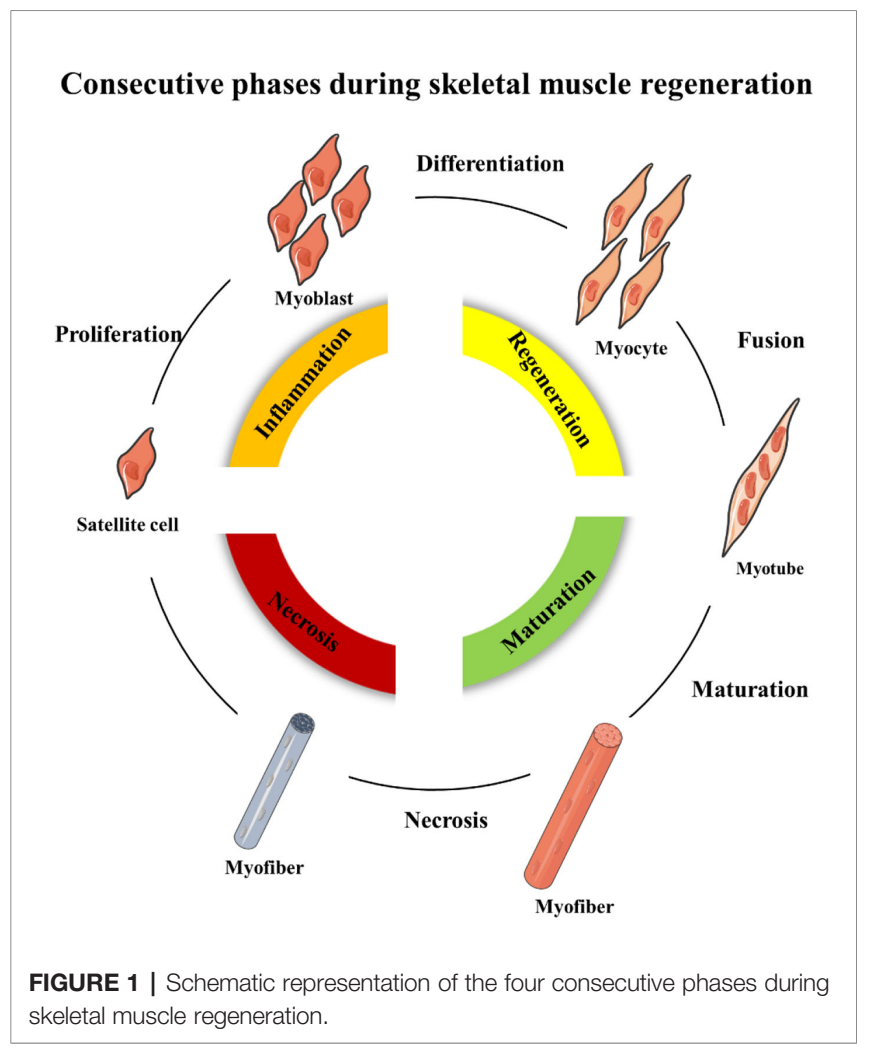

degeneration) which induce the inflammatory phase (Figure 2). The first inflammatory cells, neutrophils, are recruited to the damaged skeletal muscle within $6 \mathrm{~h}$. Soon afterwards, macrophages begin to infiltrate the damaged skeletal muscle. Similar to the inflammation in other post injury parts, the infiltrating macrophages include M1 macrophages $\left(\mathrm{CD}^{2} 8^{+} /\right.$ $\mathrm{CD} 163^{-}$) and $\mathrm{M} 2$ macrophages $\left(\mathrm{CD}^{-} 8^{-} / \mathrm{CD}_{163}{ }^{+}\right)$. In the first $24 \mathrm{~h}$, the M1 macrophages secrete pro-inflammatory cytokines such as TNF $\alpha$ and IL-1. Then, the M2 macrophages secrete antiinflammatory cytokines such as IL-10 to attenuate the inflammatory response and promote satellite cells (SC) proliferation and differentiation. (Saini et al., 2016). In the third phase, unactivated satellite cells migrate to the target location and begin to differentiate into rapidly proliferating cells, myogenic progenitor cells (Souza et al., 2018). In this phase, several musclerelated proteins are detected with highly expression such as MyoD, Desmin, and Myf5 (Zammit, 2017; Schiaffino et al., 2018). In the maturation phase, myogenic progenitor cells differentiate into myocytes and newly myofibers occur. Meanwhile, devMHC (developmental myosin heavy chain), are detected with highly expression in this phase (Sutcu and Ricchetti, 2018). At the end of the maturation phase, the nuclei move towards the edge of newly myofibers which simultaneously increase in size (Ruparelia et al., 2019). However, when ageing occurs, the process of skeletal muscle regeneration is hindered. The main obstacles include changes in the satellite cells themselves and changes in the muscle regeneration microenvironment.

\section{Changes in the Ageing Satellite Cells}

Satellite cells, also called muscle stem cells, are necessary for muscle regeneration and support the repair and remodeling of muscle fibers and help maintain healthy muscle mass throughout life. With ageing, satellite cells become losing self-renewal and regenerative capacity. The number of muscle stem cells expressing high level of transcription factor Pax7 (Pax7Hi) decreased during ageing ( $\mathrm{Li} \mathrm{H}$. et al., 2019). Recent studies have explored changes in satellite cells from the genetic and molecular levels. Epigenetic stress response in satellite cells

\section{Inflammatory Responses}

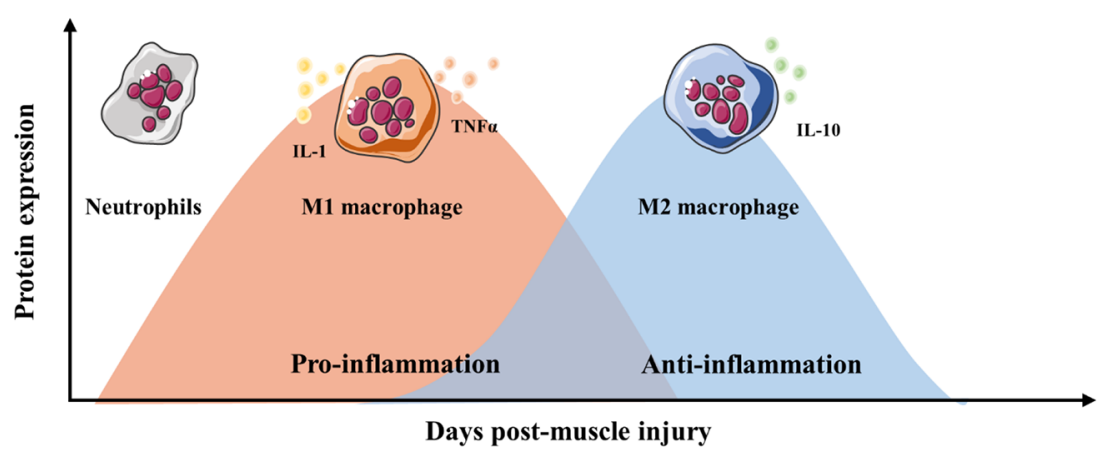

FIGURE 2 | Timeline of inflammatory responses and immune cell during muscle regeneration. 
would limit muscle regeneration by Hoxa9 developmental signaling. After analyzing the whole genome of satellite cells from healthy individuals of different ages, somatic mutagenesis was identified as another age-related negative regulator (Schwörer et al., 2016; Franco et al., 2018). Several signaling pathways were found become aberrant during muscle ageing including RTK signaling, FGF signaling and p38 MAPK (Patel et al., 2019; Bae et al., 2020). Cdon-deficit satellite cells had impaired proliferation and lower regenerative capacity via aberrant integrin and FGFR signaling. In ageing satellite cells, the p38 $\alpha / \beta$-MAPK signaling can activate high expression of p16 ${ }^{\text {Ink4a }}$ which could inhibit satellite cells self-renew (Almada and Wagers, 2016; Zhu et al., 2019). Emerging studies found that ageing leads to the asymmetric division of satellite cells (GarciaPrat and Munoz-Canoves, 2017; Feige et al., 2018; Hwang and Brack, 2018). Garcia-Prat et al. found that autophagic activity is also impaired in aged satellite cells, resulting in the accumulation of impaired proteins and mitochondria, leading to the dysfunction and reduced number of satellite cells (García-Prat et al., 2016). Oxidative stressors, especially reactive oxygen species (ROS), have been thought to have a negative effect on skeletal muscle (Baraibar et al., 2013). The oxidative capacity of skeletal muscles is closely related to general physical performance. Overexpressed ROS cause oxidative stress and mitochondrial dysfunction in cells, which eventually induce apoptosis (Zhou et al., 2018; Szentesi et al., 2019).

\section{Changes in the Aged Muscle Microenvironment}

During the ageing process, the microenvironment of muscle tissue changes, which affects the process of self-repair. Immune regulation is closely related to muscle regeneration. The numbers of myeloid lineage cells in their skeletal muscles increased during ageing, including macrophages and granulocytes (Li et al., 2020). When immune cells and factors change in the muscle microenvironment, muscle regeneration will be affected (Livshits and Kalinkovich, 2019). Immune senescence includes a systemic, chronic, low-grade pro-inflammatory state which is also known as inflammageing (Wilson et al., 2017). The proportion of serum inflammatory cytokines begins to change. The proportion of pro-inflammatory cytokines, such as IL-1 $\beta$, IL- 6 and $\mathrm{TNF} \alpha$, are gradually increasing. In contrast, the proportion of anti-inflammatory cytokines are gradually decreased (Rezus et al., 2020). The reasons for inflammageing are complex, including changes in monocyte and lymphocyte phenotypes. Meanwhile, obesity is also involved in the process of immuneageing, in which the expression of pro-inflammatory factors such as leptin increases and the expression of antiinflammatory factors such as adiponectin decreases (Wilson et al., 2017; Weyh et al., 2020). Lukjanenko L et al. found that fibro-adipogenic progenitors indirectly affect the functions of satellite cells during ageing. WNT1 Inducible Signaling Pathway Protein 1 (WISP1) secreted by fibro-adipogenic progenitors was a key matricellular communicator to repair regenerative capacity (Lukjanenko et al., 2019).

\section{POTENTIAL THERAPEUTIC TARGETS OF AGEING SKELETAL MUSCLE}

Currently, the only validated treatment of aged skeletal muscle is exercise, which can reverse different types of muscle ageing to some extent. However, for patients undergoing long-term bed rest or with other clinical complications, they are generally not recommended to exercise. Therefore, there is a need to develop new treatments for old patients to reduce skeletal muscle loss and restore muscle function. (Cohen et al., 2015). Many studies have identified various biological pathways and targets that may promote skeletal muscle regeneration, and some drugs have been tested in clinical trials. For example, A phase 2 randomized study involved 170 aged women diagnosed with sarcopenia and moderate physical dysfunction showed a significant increase in lean body mass but fail to improve muscle strength or function after receiving MK-0773, the selective androgen receptor modulator (SARM) (Papanicolaou et al., 2013). Another cohort involved in 400 individuals aged $\geq 50$ years treated with total hip arthroplasty for osteoarthritis. After receiving LY2495655, a humanized monoclonal antibody targeting myostatin, no meaningful difference in muscle strength, physical performance was found compared to placebo (Woodhouse et al., 2016).To date, results from trials have shown less success for improving muscle strength or patient physical function (Rooks and Roubenoff, 2019). As research continues, new targets have emerged in recent years, giving prospect for disease treatment. Here, we summarize the latest promising targets for treating muscle ageing.

\section{Anti-Myostatin}

Myostatin (MSTN), functioning as an endogenous regulator for the skeletal muscle growth, has drawn wide attention as a novel target. Myostatin can combine with activin receptors type IIB (ActRIIB) on the myofibers membrane which regulate skeletal muscle mass and function (White and LeBrasseur, 2014). Myostatin may also directly affect skeletal muscle mass by suppressing muscle regeneration (Ge et al., 2020). Because myostatin significantly regulates muscle regeneration, it has become a promising target for preventing the decline and dysfunction of skeletal muscle function for many years (Polkey et al., 2019). Several clinical trials have shown positive results through direct inhibiting the expression of myostatin. LY2495655, a humanized MSTN antibody, could increase appendicular lean mass in a randomised, phase 2 clinical trial (Becker et al., 2015). Bimagrumab is another human monoclonal antibody which targets activin type II receptors (ActRII). A 24-week, randomized, double-blind study, which involved 40 individuals, showed that treat with bimagrumab over 16 weeks could increase muscle mass and strength in older adults with sarcopenia. However, a multicentre, double-blind, placebo-controlled study, which involved 251 individuals, evaluated the safety and efficacy of intravenous bimagrumab in inclusion body myositis. The results indicated the treatment of bimagrumab did not meet the primary endpoint as no significant difference between bimagrumab and placebo in 6-min walking distance (6MWD) (Furrer and Handschin, 2019; Hanna et al., 2019; Hardee and Lynch, 2019). 
The above clinical research shows that large-scale multi-center clinical research is still needed to verify the efficacy and adaptive diseases of anti-myostatin treatment.

\section{Anti-Inflammatory Cytokine}

Since the immune cells are deeply involved in skeletal reconstruction process, the immune cells and inflammatory factors may become the target of improving aged skeletal muscle. Huang, $\mathrm{S}$ et al. observed the highly expression of TNF$\alpha$ and decreased expression of IL-10 in soleus muscle after treating with doxorubicin, an anti-tumor drug which could cause skeletal muscle loss in cancer patients. More importantly, doxorubicin may cause the absent of M1 macrophage during the inflammatory phase (Huang S. et al., 2017). Lee, C. et al. found Magnolol, a kind of anti-cancer drug, could attenuate the imbalance of M1/M2c macrophages to inhibit muscle loss (Lee et al., 2020). Further, Liao, Z.H., et al. found that regulating the functions of Treg cells would also be an important measure to promote muscle regeneration. They treated cardiotoxin (CTX)induced muscle injury by estrogen and the results indicated estrogen could suppress immune response and reverse phenotypes of monocytes/macrophages by regulating the function of Treg cells, and suppressing Th1 response in the inflammatory phase (Liao et al., 2019). Administration of exogenous anti-inflammatory factors may also improve skeletal muscle regeneration. Costamagna, $\mathrm{D}$ et al. found the IL-4 treatment would improve the performances and prolonged survival of colon carcinoma-bearing (C26) mice. IL-4 could rescue muscle mass by increasing protein synthesis and promote myogenesis (Costamagna et al., 2020). Du $\mathrm{H}$ et al. investigated that overexpression of ADAMTS1 in macrophages could active satellite cell and promote muscle regeneration by reduces Notch signaling (Du et al., 2017). Although a large number of studies have suggested that immune cells and immune factors play an important role in promoting muscle regeneration, the role of the immune system in ageing muscle loss is unknown. Therefore, further research is needed to further explore the immune microenvironment around ageing skeletal muscles for possible therapeutic targets.

\section{Nucleic Acids}

Nucleic acids, particularly non-encoding nucleic acid, act as a key modulator of skeletal muscle proliferation and regeneration. It is noteworthy that a group of muscle- specific miRNAs, myomiRNAs, are closely related to skeletal muscle development and regeneration, including miR-133, miR-206, miR-208b, and miR-499. Iannone, F et al. investigated that the under-expression of miR-133b may contribute to the impaired regenerative capacity of satellite cells (Iannone et al., 2020). The expression of miR-206 increased during muscle regeneration result in the inhibition of Pax3, Pax7, and cMet. These downstream genes subsequently affect myogenic differentiation (McCarthy, 2008).

As EVs are closely related to exercise, researchers have confirmed that different types of exercise would release a large number of EVs into the blood circulation. Since the discovery of
EVs can transfer functional non-encoding nucleic acid, EVassociated nucleic acid may play a key role in muscle cell-cell communication (Yin et al., 2018). Fulzele, S. et al. found a significant increase in expression of senescence-associated EVsderived miR-34a in skeletal muscle from ageing mice. The results indicated that senescence-associated EVs secreted from skeletal muscle may directly reduce stem cell populations via their microRNA cargo (Fulzele et al., 2019). As the growing understanding of EVs, more therapeutic targets may be constantly discovered.

\section{Bone-Derived Factors}

Bone and muscle are closely related, and many studies have found that bone and muscle interact on each other. As emerging evidence supports the concept that bone would act as an endocrine organ, bone-secreted factors may be an important target for muscle regeneration (Figure 3). Osteocalcin (OCN) is currently the most widely studied bone-derived factor. OCN regulates the uptake and catabolism of nutrients in muscle during exercise by promoting the release of interleukin- 6 from myofibers. More importantly, the muscle mass increased significantly after giving exogenous OCN in older mice. These findings strongly suggest that OCN would be a promising therapeutic target for ageing muscle regeneration(Mera et al., 2016a; Mera et al., 2016b). In contrast, transforming growth factor (TGF)- $\beta$ derived from bone surface was a negative regulator which contributes to muscle weakness (Waning et al., 2015). Sclerostin is mainly secreted by osteocytes and osteoblasts which is a key inhibitor of Wnt signaling. Sclerostin could inhibit myogenic differentiation by activating Wnt/ $\beta$ catenin pathway in vitro experiment. Hesse $\mathrm{E}$ et al. found that sclerostin antibody would alleviate muscle weakness and improve skeletal muscle function (Huang J. et al., 2017; Hesse et al., 2019). The receptor-activator of nuclear factor $\kappa \mathrm{B} /$ receptor-activator of nuclear factor $\kappa \mathrm{B}$ ligand/osteoprotegerin (RANK/RANKL/OPG) is a key pathway for bone remodeling. Recent studies found that bone derived RANKL inhibitor could significantly improve skeletal muscle function and restore bone mass. OPG-deficient mice displayed reduced muscle weakness with selective atrophy of fast-twitch-type IIb myofibers. After using RANKL inhibitors, represented by Denosumab, patients have improved handgrip strength which indicate RANKL inhibitors may be an important method for treating muscle diseases (Dufresne et al., 2018; Bonnet et al., 2019; Hamoudi et al., 2020).

Although there are many therapeutic targets for ageing skeletal muscle, a very important point is how to accurately deliver functional nucleic acids or proteins. Obviously, the current clinical trials for muscle regeneration have encountered some difficulties. For example, the anti-myostatin drug has not achieved the expected effect. The results of clinical trials suggest that the exploration of functional molecules may not achieve good therapeutic effects. How to accurately deliver and release functional molecules may be another important issue to be explored. We believe that building an appropriate delivery system for muscle regeneration is another area worthy of in-depth study. 


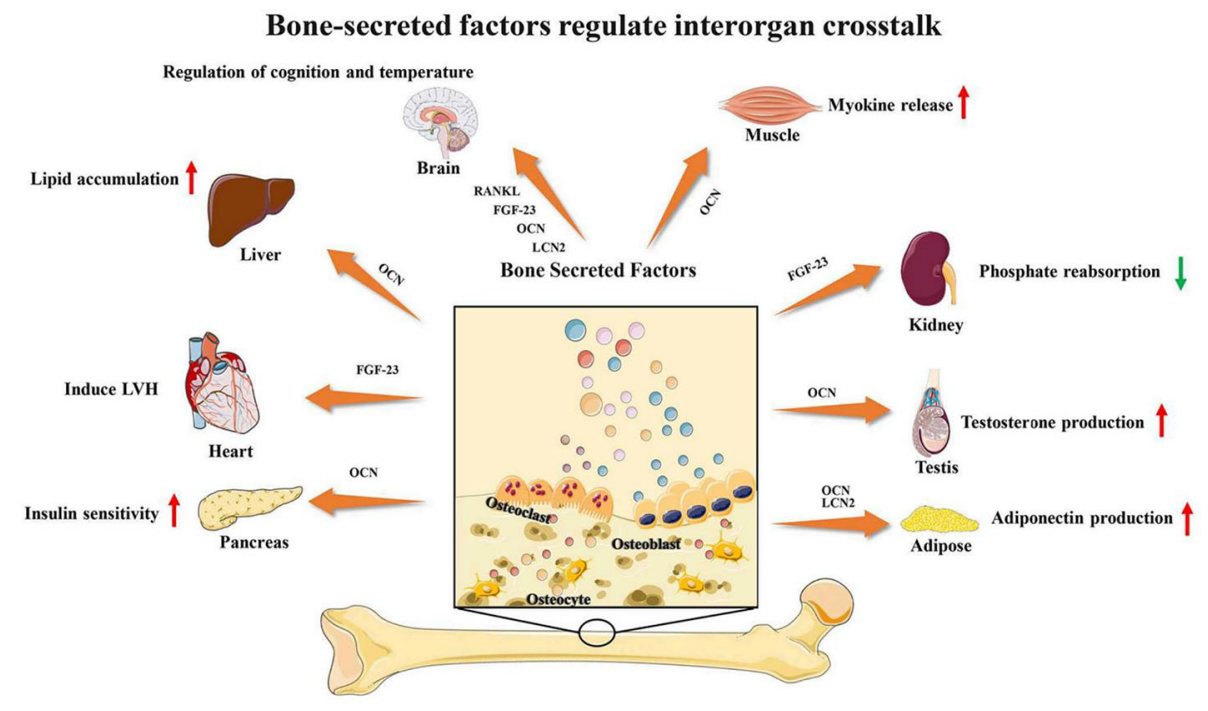

FIGURE 3 | Bone-secreted factors regulate interorgan crosstalk. Reproduced, with permission, from (Li Y. et al., 2019).

\section{DRUG DELIVERY SYSTEM FOR AGEING SKELETAL MUSCLE REGENERATION}

To treat aged skeletal muscle dysfunction, appropriate drug delivery system that loads with potential therapeutic factors would be highly desired. Here, we summarize the current promising drug delivery systems, and future studies may load different active factors on the basis of these systems.

\section{Adeno-Associated Virus (AAV)}

Adeno-associated viral (AAV) vectors are currently developing rapidly in the treatment of gene-related muscle disorders. Natural or engineered viral capsids have been successfully constructed by transferring nucleic acids, antibodies or geneediting machinery via various routes of administration successfully (Lisowski et al., 2015; Naso et al., 2017). Currently, AAV appears to be the most promising vector for gene therapy, especially the treatment of Duchenne muscular dystrophy and Spinal muscular atrophy (SMA) (Groen et al., 2018; Verhaart and Aartsma-Rus, 2019). There will be more clinical trials to be performed in the future, and it will also be applied to other muscle diseases. However, the limitations of AAV are also obvious. Lacking muscle cell tropism induces the inefficiency of AAV. Lacking effective nucleic acid editing targets also makes AAV difficult to use as an effective means to promote ageing skeletal muscle regeneration. Therefore, we propose constructing novel drug delivery systems to repair skeletal muscles with complex pathogenesis.

\section{Muscle-Targeting Delivery System}

Because ageing-related muscle loss has no local lesions, drug therapy can only be administered systemically. The problem with systemic administration is the side effects in other organs, and the high concentration in liver and kidney. To solve above problems, another important strategy for building drug delivery systems is muscle-targeting delivery systems. A muscle-targeting delivery system requires a targeted motif, which can be combined with a delivery system to specifically deliver drugs to skeletal muscles. For example, skeletal musclerelated cell surface-specific proteins can serve as a targeted motif. Large molecules such as antibodies or antibody-drug conjugates would also be a potential method of targeting (Ebner et al., 2015). Several examples of muscle-targeting peptide have been reported. Samoylova TI et al. found a heptapeptide sequence, ASSLNIA, which could improve specificity for binding to skeletal muscle by screening a random phage display library (Samoylova and Smith, 1999). Then, Jativa S D et al. develop a generation 5polyamidoamine dendrimer (G5-PAMAM) modified with ASSLNIA to synergistically enhance gene delivery to skeletal muscle cells (Jativa et al., 2019). Gao X et al. identified a novel 12mer peptide (M12) which was shown more effective musclehoming ability. This motif could enhance binding affinity to myoblast in vitro. Conjugated muscle-homing peptide with phosphorodiamidate morpholino oligomers showed significant improvement in grip strength in $\mathrm{mdx}$ mice (Seow et al., 2010; Gao et al., 2014). Currently, only a few studies have discovered molecules with muscle-targeting functions. Therefore, subsequent research still needs to explore more novel muscletargeting molecules and make appropriate modifications to ameliorate ageing-related muscle loss.

\section{Nanoparticles}

The growing concern of nanoparticles is triggered by the rapid development of nanotechnology. Nanoparticles have many unique advantages including enhanced tissue targeting, nucleic acids protection from degradation, and low immunogenicity 
(Nance et al., 2018; Nag et al., 2020). For example, Poussard, S et al. observed the silica nanoparticles uptake by myoblasts and promote the differentiation in vitro (Poussard et al., 2015). Ge. J et al. developed a kind of monodispersed gold and goldsilver nanoparticles (AuNPs and Au-AgNPs) which could support adhesion and proliferation of myoblast by motivating the p38 $\alpha$ MAPK signaling pathway (Ge et al., 2018). Raimondo, T.M. et al. found that constructing cytokines binding gold nanoparticles could regulate the inflammatory phase by changing M2 macrophage polarization, which subsequently promote regeneration and increased newly myofibles function (Raimondo and Mooney, 2018). Nanoparticles are easy to prepare on a uniform scale and are easier to modify. There are many optional materials for nanoparticles such as metal particles or silica particles. However, the follow-up research still needs to consider the safety of nanoparticles, including material toxicity, dose toxicity, and metabolic rate. In addition, how to increase the skeletal muscle targeting of nanoparticles still needs to be explored.

\section{Extracellular Vesicles (EVs)}

EVs, 30-150 nm in diameter, have been demonstrated to transfer functional proteins, nucleic acid (e.g. mRNA, miRNA, lncRNA) to neighboring or remote cells. The membrane of EVs acts as a natural barrier to prevent degradation in the blood circulation (Figure 4). EVs can be administered via intravenous (IV) and intranasal routes to reach the target location, depending on the therapeutic purpose. Due to these characteristics, EVs are considered ideal natural systems for drug delivery (Vader et al., 2016; Skotland et al., 2020; Rong et al., 2020).
Many studies show that EVs are closely involved in skeletal muscle metabolism and regeneration. Zanotti $S$ et al. found that EVs derived from local fibroblasts in the Duchenne muscular dystrophy (DMD) muscle are able to induce a transfer of unactivated fibroblasts to myofibroblasts thereby motivating the fibrotic response (Zanotti et al., 2018). As mentioned above, Fulzele, S. et al. found EVs-derived miR-34a could regulate muscle metabolism (Fulzele et al., 2019). Many studies investigated that physical exercise, which is closely related with muscle regeneration, can induce rapid release of EVs into the blood circulation. Fruhbeis, $\mathrm{C}$ et al. found that a consecutive release of EVs would be triggered after physical exercise. During physical exercise, the released-EVs may participate in intercellular communication or remote communication which involve signaling across tissues and organs (Frühbeis et al., 2015). Guescini, $M$ found similar results and further showed that muscle-specific miRNAs in EVs were significantly upregulated (Guescini et al., 2015). Whitham, $M$ et al. found that exerciserelated EVs tend to accumulate in the liver and can transfer inner proteins (Whitham et al., 2018). These studies have demonstrated the promising potential of EVs for therapeutic drug delivery in various muscle diseases. It is worth noting that Ran $\mathrm{N}$ et al. created a novel exosome-based delivery system by anchoring the inhibitory domain of myostatin propeptide. The exosomedelivery system could efficiently promote muscle function in mdx mice (Ran et al., 2020). Gao et al. also demonstrated a muscle-targeting exosome-based delivery system, which was modified by CP05, could increase dystrophin expression in $\mathrm{mdx}$ mice (Gao et al., 2018). These exosome-based delivery platforms may be effective delivery methods for the treatment of DMD.

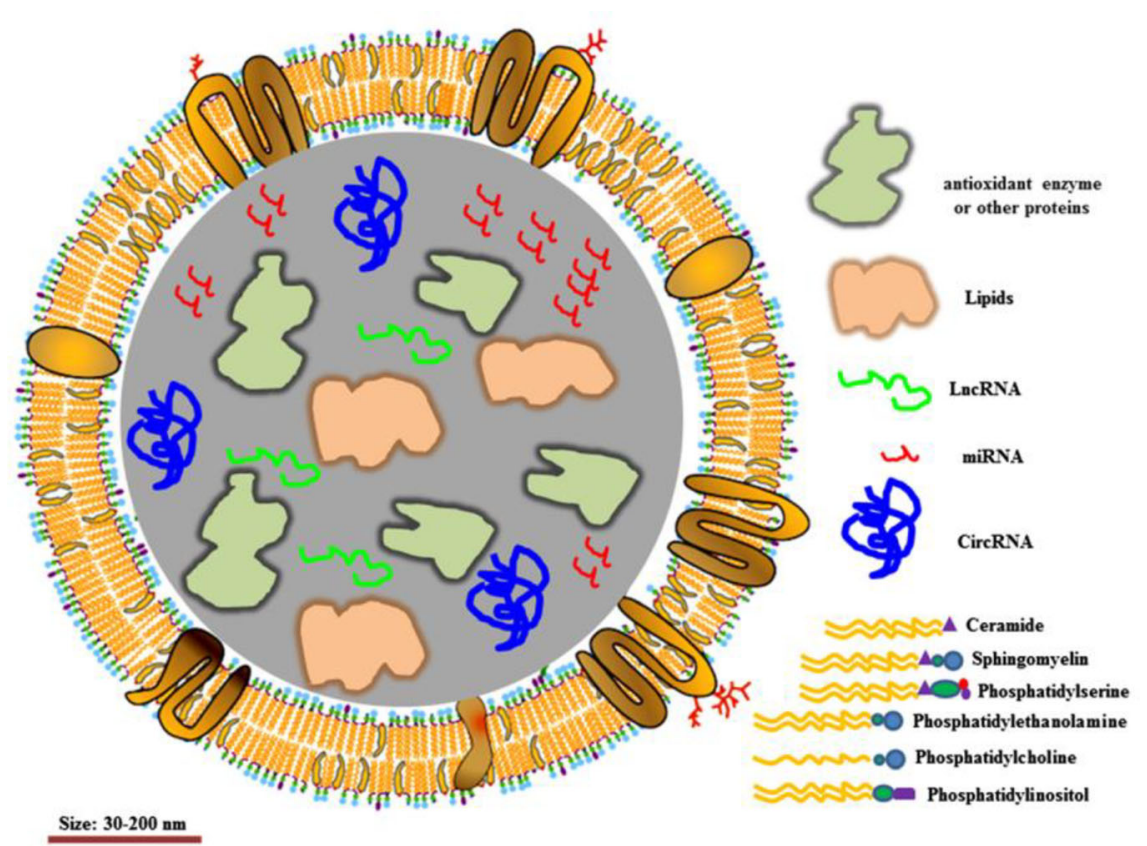

FIGURE 4 | Structure of extracellular vesicles. Reproduced, with permission, from (Rong et al., 2020). 
The advantages of exosomes are good biological safety, easy absorption and metabolism. Its widespread presence in the body also makes it relatively easy to obtain. It has been found that exosomes have a natural targeting function, which makes it an ideal precision delivery system (Song et al., 2019). However, the research on exosomes is still in an early stage, and the following issues still need to be solved. First, constructing a large number of exosomes with uniform size is still difficult. Efficient loading rate also needs to be considered. In addition, the selection of cell-derived exosomes to construct a delivery system still requires further exploration. At present, there are few studies on the affinity and targeting ability of different exosomes to skeletal muscle. When the problem of exosome preparation is solved, screening the appropriate functional molecule is another key step. In summary, exosomes may be an ideal delivery system for aging skeletal muscle regeneration. Exploring the characteristics and modification of exosomes still needs further investigation.

\section{CONCLUSION AND FUTURE DIRECTIONS}

Currently there is no ideal treatment for the ageing-related loss of skeletal muscle. Nano-carrier drug delivery system is an emerging area which may provide the ultimate solution for the clinical treatment. Future research should be divided into two aspects for further exploration. Current studies on potential

\section{REFERENCES}

Almada, A. E., and Wagers, A. J. (2016). Molecular circuitry of stem cell fate in skeletal muscle regeneration, ageing and disease. Nat. Rev. Mol. Cell Biol. 17 (5), 267-279. doi: 10.1038/nrm.2016.7

Bae, J. H., Hong, M., Jeong, H. J., Kim, H., Lee, S. J., Ryu, D., et al. (2020). Satellite cell-specific ablation of Cdon impairs integrin activation, FGF signalling, and muscle regeneration. J. Cachexia Sarcopenia Muscle. doi: 10.1002/jcsm.12563

Baraibar, M. A., Gueugneau, M., Duguez, S., Butler-Browne, G., Bechet, D., and Friguet, B. (2013). Expression and modification proteomics during skeletal muscle ageing. Biogerontology 14 (3), 339-352. doi: 10.1007/s10522-013-9426-7

Barberi, L., Scicchitano, B. M., De Rossi, M., Bigot, A., Duguez, S., Wielgosik, A., et al. (2013). Age-dependent alteration in muscle regeneration: the critical role of tissue niche. Biogerontology 14 (3), 273-292. doi: 10.1007/s10522-013-9429-4

Becker, C., Lord, S. R., Studenski, S. A., Warden, S. J., Fielding, R. A., Recknor, C. P., et al. (2015). Myostatin antibody (LY2495655) in older weak fallers: a proof-of-concept, randomised, phase 2 trial. Lancet Diabetes Endocrinol. 3 (12), 948-957. doi: 10.1016/S2213-8587(15)00298-3

Bonnet, N., Bourgoin, L., Biver, E., Douni, E., and Ferrari, S. (2019). RANKL inhibition improves muscle strength and insulin sensitivity and restores bone mass. J. Clin. Invest. 129 (8), 3214-3223. doi: 10.1172/JCI125915

Cohen, S., Nathan, J. A., and Goldberg, A. L. (2015). Muscle wasting in disease: molecular mechanisms and promising therapies. Nat. Rev. Drug Discovery 14 (1), 58-74. doi: 10.1038/nrd4467

Costamagna, D., Duelen, R., Penna, F., Neumann, D., Costelli, P., and Sampaolesi, M. (2020). Interleukin-4 administration improves muscle function, adult myogenesis, and lifespan of colon carcinoma-bearing mice. $J$. Cachexia Sarcopenia Muscle 11 (3), 783-801. doi: 10.1002/jcsm.12539

Cuesta-Triana, F., Verdejo-Bravo, C., Fernandez-Perez, C., and Martin-Sanchez, F. J. (2019). Effect of Milk and Other Dairy Products on the Risk of Frailty, Sarcopenia, and Cognitive Performance Decline in the Elderly: A Systematic Review. Adv. Nutr. 10 (suppl_2), S105-S119. doi: 10.1093/advances/nmy105 targets for skeletal muscle regeneration do not consider the characteristics of aging. Taking into account the changes in the muscle satellite cells and immune microenvironment in aging skeletal muscle, new therapeutic targets may be discovered. Although the nanocarrier delivery system provides a good method for the treatment of aging diseases, many safety and effectiveness issues still need to be explored. The construction of the delivery system should be carried out simultaneously with the research on the mechanism of regulating muscle regeneration. The ideal delivery system should not only play a role in promoting, but adopt multiple control methods to maximize the improvement of aging muscle loss.

\section{AUTHOR CONTRIBUTIONS}

PY, LZ, and PT made major contributions to the conception of the work. YL, MC, and YY drafted the manuscript. YZ, ML, YQ, and SC revised the manuscript.

\section{ACKNOWLEDGMENTS}

This work was supported by National Natural Science Foundation of China (81702176, 81772369, 81972115 and 51803188).

Du, H., Shih, C. H., Wosczyna, M. N., Mueller, A. A., Cho, J., Aggarwal, A., et al. (2017). Macrophage-released ADAMTS1 promotes muscle stem cell activation. Nat. Commun. 8 (1), 669. doi: 10.1038/s41467-017-00522-7

Dufresne, S. S., Boulanger-Piette, A., Bosse, S., Argaw, A., Hamoudi, D., Marcadet, L., et al. (2018). Genetic deletion of muscle RANK or selective inhibition of RANKL is not as effective as full-length OPG-fc in mitigating muscular dystrophy. Acta Neuropathol. Commun. 6 (1), 31. doi: 10.1186/s40478-0180533-1

Ebner, D. C., Bialek, P., El-Kattan, A. F., Ambler, C. M., and Tu, M. (2015). Strategies for skeletal muscle targeting in drug discovery. Curr. Pharm. Des. 21 (10), 1327-1336. doi: 10.2174/1381612820666140929095755

Fan, W., Atkins, A. R., Yu, R. T., Downes, M., and Evans, R. M. (2013). Road to exercise mimetics: targeting nuclear receptors in skeletal muscle. J. Mol. Endocrinol. 51 (3), T87-T100. doi: 10.1530/JME-13-0258

Feige, P., Brun, C. E., Ritso, M., and Rudnicki, M. A. (2018). Orienting Muscle Stem Cells for Regeneration in Homeostasis, Aging, and Disease. Cell Stem Cell 23 (5), 653-664. doi: 10.1016/j.stem.2018.10.006

Frühbeis, C., Helmig, S., Tug, S., Simon, P., and Krämer-Albers, E. (2015). Physical exercise induces rapid release of small extracellular vesicles into the circulation. J. Extracell. Vesicles 4 (1), 28211-28239. doi: 10.3402/jev.v4.28239

Franco, I., Johansson, A., Olsson, K., Vrtačnik, P., Lundin, P., Helgadottir, H. T., et al. (2018). Somatic mutagenesis in satellite cells associates with human skeletal muscle aging. Nat. Commun. 9 (1), 800. doi: 10.1038/s41467-018-03244-6

Fulzele, S., Mendhe, B., Khayrullin, A., Johnson, M., Kaiser, H., Liu, Y., et al. (2019). Muscle-derived miR-34a increases with age in circulating extracellular vesicles and induces senescence of bone marrow stem cells. Aging (Albany NY) 11 (6), 1791-1803. doi: 10.18632/aging.101874

Furrer, R., and Handschin, C. (2019). Muscle Wasting Diseases: Novel Targets and Treatments. Annu. Rev. Pharmacol. Toxicol. 59, 315-339. doi: 10.1146/ annurev-pharmtox-010818-021041

Gao, X., Zhao, J., Han, G., Zhang, Y., Dong, X., Cao, L., et al. (2014). Effective dystrophin restoration by a novel muscle-homing peptide-morpholino 
conjugate in dystrophin-deficient mdx mice. Mol. Ther. 22 (7), 1333-1341. doi: $10.1038 / \mathrm{mt} .2014 .63$

Gao, X., Ran, N., Dong, X., Zuo, B., Yang, R., Zhou, Q., et al. (2018). Anchor peptide captures, targets, and loads exosomes of diverse origins for diagnostics and therapy. Sci. Trans. Med. 10 (444), t195. doi: 10.1126/scitranslmed.aat0195

García-Prat, L., Martínez-Vicente, M., Perdiguero, E., Ortet, L., RodríguezUbreva, J., Rebollo, E., et al. (2016). Autophagy maintains stemness by preventing senescence. Nature 529 (7584), 37-42. doi: 10.1038/nature16187

Garcia-Prat, L., and Munoz-Canoves, P. (2017). Aging, metabolism and stem cells: Spotlight on muscle stem cells. Mol. Cell Endocrinol. 445, 109-117. doi: 10.1016/j.mce.2016.08.021

Ge, J., Liu, K., Niu, W., Chen, M., Wang, M., Xue, Y., et al. (2018). Gold and goldsilver alloy nanoparticles enhance the myogenic differentiation of myoblasts through p38 MAPK signaling pathway and promote in vivo skeletal muscle regeneration. Biomaterials 175, 19-29. doi: 10.1016/j.biomaterials.2018.05.027

Ge, L., Dong, X., Gong, X., Kang, J., Zhang, Y., and Quan, F. (2020). Mutation in myostatin 3'UTR promotes $\mathrm{C} 2 \mathrm{C} 12$ myoblast proliferation and differentiation by blocking the translation of MSTN. Int. J. Biol. Macromol. 154, 634-643. doi: 10.1016/j.ijbiomac.2020.03.043

Groen, E. J. N., Talbot, K., and Gillingwater, T. H. (2018). Advances in therapy for spinal muscular atrophy: promises and challenges. Nat. Rev. Neurol. 14 (4), 214-224. doi: 10.1038/nrneurol.2018.4

Guescini, M., Canonico, B., Lucertini, F., Maggio, S., Annibalini, G., Barbieri, E., et al. (2015). Muscle Releases Alpha-Sarcoglycan Positive Extracellular Vesicles Carrying miRNAs in the Bloodstream. PloS One 10 (5), e125094. doi: 10.1371/ journal.pone.0125094

Hamoudi, D., Bouredji, Z., Marcadet, L., Yagita, H., Landry, L. B., Argaw, A., et al. (2020). Muscle weakness and selective muscle atrophy in osteoprotegerindeficient mice. Hum. Mol. Genet. 29 (3), 483-494. doi: 10.1093/hmg/ddz312

Hanna, M. G., Badrising, U. A., Benveniste, O., Lloyd, T. E., Needham, M., Chinoy, H., et al. (2019). Safety and efficacy of intravenous bimagrumab in inclusion body myositis (RESILIENT): a randomised, double-blind, placebocontrolled phase 2b trial. Lancet Neurol. 18 (9), 834-844. doi: 10.1016/S14744422(19)30200-5

Hardee, J. P., and Lynch, G. S. (2019). Current pharmacotherapies for sarcopenia. Expert Opin. Pharmacother. 20 (13), 1645-1657. doi: 10.1080/ 14656566.2019.1622093

Hesse, E., Schroder, S., Brandt, D., Pamperin, J., Saito, H., and Taipaleenmaki, H. (2019). Sclerostin inhibition alleviates breast cancer-induced bone metastases and muscle weakness. JCI Insight 5 (9), e125543. doi: 10.1172/ jci.insight. 125543

Huang, J., Romero-Suarez, S., Lara, N., Mo, C., Kaja, S., Brotto, L., et al. (2017). Crosstalk between MLO-Y4 osteocytes and C2C12 muscle cells is mediated by the Wnt/beta-catenin pathway. JBMR Plus 1 (2), 86-100. doi: 10.1002/ jbm4.10015

Huang, S., Wu, J., Saovieng, S., Chien, W., Hsu, M., Li, X., et al. (2017). Doxorubicin inhibits muscle inflammation after eccentric exercise. J. Cachexia Sarcopenia Muscle 8 (2), 277-284. doi: 10.1002/jcsm. 12148

Hwang, A. B., and Brack, A. S. (2018). Muscle Stem Cells and Aging. Curr. Top. Dev. Biol. 126, 299-322. doi: 10.1016/bs.ctdb.2017.08.008

Iannone, F., Montesanto, A., Cione, E., Crocco, P., Caroleo, M. C., Dato, S., et al. (2020). Expression Patterns of Muscle-Specific miR-133b and miR-206 Correlate with Nutritional Status and Sarcopenia. Nutrients 12 (2), 297. doi: 10.3390/nu12020297

Jativa, S. D., Thapar, N., Broyles, D., Dikici, E., Daftarian, P., Jiménez, J. J., et al. (2019). Enhanced Delivery of Plasmid DNA to Skeletal Muscle Cells using a DLC8-Binding Peptide and ASSLNIA-Modified PAMAM Dendrimer. Mol. Pharm. 16 (6), 2376-2384. doi: 10.1021/acs.molpharmaceut.8b01313

Lee, C., Jeong, H., Lee, H., Hong, M., Park, S., and Bae, H. (2020). Magnolol Attenuates Cisplatin-Induced Muscle Wasting by M2c Macrophage Activation. Front. Immunol. 11, 77. doi: 10.3389/fimmu.2020.00077

Li, H., Chen, Q., Li, C., Zhong, R., Zhao, Y., Zhang, Q., et al. (2019). Musclesecreted granulocyte colony-stimulating factor functions as metabolic niche factor ameliorating loss of muscle stem cells in aged mice. EMBO J. 38 (24), e102154. doi: 10.15252/embj.2019102154

Li, Y., Yin, P., Guo, Z., Lv, H., Deng, Y., Chen, M., et al. (2019). Bone-Derived Extracellular Vesicles: Novel Players of Interorgan Crosstalk. Front. Endocrinol. 10, 846. doi: 10.3389/fendo.2019.00846
Li, J., Yi, X., Yao, Z., Chakkalakal, J. V., Xing, L., and Boyce, B. F. (2020). TNF receptor-associated factor 6 mediates $\mathrm{TNF} \alpha$-induced skeletal muscle atrophy in mice during aging. J. Bone Miner. Res. doi: 10.1002/jbmr.4021

Liao, Z. H., Huang, T., Xiao, J. W., Gu, R. C., Ouyang, J., Wu, G., et al. (2019). Estrogen signaling effects on muscle-specific immune responses through controlling the recruitment and function of macrophages and $\mathrm{T}$ cells. Skeletal Muscle 9 (1), 20. doi: 10.1186/s13395-019-0205-2

Lisowski, L., Tay, S. S., and Alexander, I. E. (2015). Adeno-associated virus serotypes for gene therapeutics. Curr. Opin. Pharmacol. 24, 59-67. doi: 10.1016/j.coph.2015.07.006

Livshits, G., and Kalinkovich, A. (2019). Inflammaging as a common ground for the development and maintenance of sarcopenia, obesity, cardiomyopathy and dysbiosis. Ageing Res. Rev. 56, 100980. doi: 10.1016/j.arr.2019.100980

Lukjanenko, L., Karaz, S., Stuelsatz, P., Gurriaran-Rodriguez, U., Michaud, J., Dammone, G., et al. (2019). Aging Disrupts Muscle Stem Cell Function by Impairing Matricellular WISP 1 Secretion from Fibro-Adipogenic Progenitors. Cell Stem Cell 24 (3), 433-446. doi: 10.1016/j.stem.2018. 12.014

McCarthy, J. (2008). MicroRNA-206: The skeletal muscle-specific myomiR. Biochim. Biophys. Acta (BBA) - Gene Regul. Mech. 1779 (11), 682-691. doi: 10.1016/j.bbagrm.2008.03.001

McKeon-Fischer, K. D., Browe, D. P., Olabisi, R. M., and Freeman, J. W. (2015). Poly(3,4-ethylenedioxythiophene) nanoparticle and poly( $\varepsilon$-caprolactone) electrospun scaffold characterization for skeletal muscle regeneration. J. Biomed. Mater. Res. Part A 103 (11), 3633-3641. doi: 10.1002/jbm.a.35481

Mera, P., Laue, K., Ferron, M., Confavreux, C., Wei, J., Galan-Diez, M., et al. (2016a). Osteocalcin Signaling in Myofibers Is Necessary and Sufficient for Optimum Adaptation to Exercise. Cell Metab. 23 (6), 1078-1092. doi: 10.1016/ j.cmet.2016.05.004

Mera, P., Laue, K., Wei, J., Berger, J. M., and Karsenty, G. (2016b). Osteocalcin is necessary and sufficient to maintain muscle mass in older mice. Mol. Metab. 5 (10), 1042-1047. doi: 10.1016/j.molmet.2016.07.002

Nag, O. K., Muroski, M. E., Hastman, D. A., Almeida, B., Medintz, I. L., Huston, A. L., et al. (2020). Nanoparticle-Mediated Visualization and Control of Cellular Membrane Potential: Strategies, Progress, and Remaining Issues. ACS Nano. 14 (3), 2659-2677. doi: 10.1021/acsnano.9b10163

Nance, M. E., Hakim, C. H., Yang, N. N., and Duan, D. (2018). Nanotherapy for Duchenne muscular dystrophy. Wiley Interdiscip. Rev. Nanomed. Nanobiotechnol. 10 (2). doi: 10.1002/wnan.1472

Naso, M. F., Tomkowicz, B., Perry, W. R., and Strohl, W. R. (2017). AdenoAssociated Virus (AAV) as a Vector for Gene Therapy. BioDrugs 31 (4), 317334. doi: 10.1007/s40259-017-0234-5

Papanicolaou, D. A., Ather, S. N., Zhu, H., Zhou, Y., Lutkiewicz, J., Scott, B. B., et al. (2013). A phase IIA randomized, placebo-controlled clinical trial to study the efficacy and safety of the selective androgen receptor modulator (SARM), MK-0773 in female participants with sarcopenia. J. Nutr. Health Aging 17 (6), 533-543. doi: 10.1007/s12603-013-0335-x

Patel, K., Simbi, B., Ritvos, O., Vaiyapuri, S., and Dhoot, G. K. (2019). Dysregulated cell signalling and reduced satellite cell potential in ageing muscle. Exp. Cell Res. 385 (2), 111685. doi: 10.1016/j.yexcr.2019.111685

Pedersen, B. K. (2013). Muscle as a secretory organ. Compr. Physiol. 3 (3), 13371362. doi: $10.1002 /$ cphy.c120033

Polkey, M. I., Praestgaard, J., Berwick, A., Franssen, F. M. E., Singh, D., Steiner, M. C., et al. (2019). Activin Type II Receptor Blockade for Treatment of Muscle Depletion in Chronic Obstructive Pulmonary Disease. A Randomized Trial. Am. J. Respir. Crit. Care Med. 199 (3), 313-320. doi: 10.1164/rccm.2018020286OC

Poussard, S., Decossas, M., Le Bihan, O., Mornet, S., Naudin, G., and Lambert, O. (2015). Internalization and fate of silica nanoparticles in C2C12 skeletal muscle cells: evidence of a beneficial effect on myoblast fusion. Int. J. Nanomed. 10, 1479-1492. doi: 10.2147/IJN.S74158

Raimondo, T. M., and Mooney, D. J. (2018). Functional muscle recovery with nanoparticle-directed M2 macrophage polarization in mice. Proc. Natl. Acad. Sci. 115 (42), 10648-10653. doi: 10.1073/pnas. 1806908115

Ran, N., Gao, X., Dong, X., Li, J., Lin, C., Geng, M., et al. (2020). Effects of exosome-mediated delivery of myostatin propeptide on functional recovery of mdx mice. Biomaterials 236, 119826. doi: 10.1016/j.biomaterials. 2020.119826 
Rezus, E., Burlui, A., Cardoneanu, A., Rezus, C., Codreanu, C., Parvu, M., et al. (2020). Inactivity and Skeletal Muscle Metabolism: A Vicious Cycle in Old Age. Int. J. Mol. Sci. 21 (2), 592. doi: 10.3390/ijms21020592

Rong, S., Wang, L., Peng, Z., Liao, Y., Li, D., Yang, X., et al. (2020). The mechanisms and treatments for sarcopenia: could exosomes be a perspective research strategy in the future? J. Cachexia Sarcopenia Muscle 11 (2), 348-365. doi: $10.1002 /$ jcsm. 12536

Rooks, D., and Roubenoff, R. (2019). Development of Pharmacotherapies for the Treatment of Sarcopenia. J. Frailty Aging 8 (3), 120-130. doi: 10.14283/ jfa.2019.11

Ruparelia, A. A., Ratnayake, D., and Currie, P. D. (2019). Stem cells in skeletal muscle growth and regeneration in amniotes and teleosts: Emerging themes. WIREs Dev. Biol. 9 (2), e365. doi: 10.1002/wdev.365

Saini, J., McPhee, J. S., Al-Dabbagh, S., Stewart, C. E., and Al-Shanti, N. (2016). Regenerative function of immune system: Modulation of muscle stem cells. Ageing Res. Rev. 27, 67-76. doi: 10.1016/j.arr.2016.03.006

Samoylova, T. I., and Smith, B. F. (1999). Elucidation of muscle-binding peptides by phage display screening. Muscle Nerve 22 (4), 460-466. doi: 10.1002/(SICI) 1097-4598(199904)22:4<460::AID-MUS6>3.0.CO;2-L

Schiaffino, S., Dyar, K. A., and Calabria, E. (2018). Skeletal muscle mass is controlled by the MRF4-MEF2 axis. Curr. Opin. Clin. Nutr. Metab. Care 21 (3), 164-167. doi: 10.1097/MCO.0000000000000456

Schmidt, M., Schüler, S. C., Hüttner, S. S., von Eyss, B., and von Maltzahn, J. (2019). Adult stem cells at work: regenerating skeletal muscle. Cell. Mol. Life Sci. 76 (13), 2559-2570. doi: 10.1007/s00018-019-03093-6

Schwörer, S., Becker, F., Feller, C., Baig, A. H., Köber, U., Henze, H., et al. (2016). Epigenetic stress responses induce muscle stem-cell ageing by Hoxa9 developmental signals. Nature 540 (7633), 428-432. doi: 10.1038/nature20603

Seow, Y., Yin, H., and Wood, M. J. (2010). Identification of a novel muscle targeting peptide in mdx mice. Peptides 31 (10), 1873-1877. doi: 10.1016/ j.peptides.2010.06.036

Skotland, T., Sagini, K., Sandvig, K., and Llorente, A. (2020). An emerging focus on lipids in extracellular vesicles. Adv. Drug Delivery Rev. S0169-409X(20)300144. doi: 10.1016/j.addr.2020.03.002

Song, H., Li, X., Zhao, Z., Qian, J., Wang, Y., Cui, J., et al. (2019). Reversal of osteoporotic activity by endothelial cell-secreted bone targeting and biocompatible exosomes. Nano Lett. 19 (5), 3040-3048. doi: 10.1021/ acs.nanolett.9b00287

Souza, N., Mesquita-Ferrari, R. A., Rodrigues, M., Da, S. D., Ribeiro, B. G., Alves, A. N., et al. (2018). Photobiomodulation and different macrophages phenotypes during muscle tissue repair. J. Cell Mol. Med. 22 (10), 4922-4934. doi: 10.1111/jcmm.13757

Sutcu, H. H., and Ricchetti, M. (2018). Loss of heterogeneity, quiescence, and differentiation in muscle stem cells. Stem Cell Invest. 5, 9. doi: 10.21037/ sci.2018.03.02

Szentesi, P., Csernoch, L., Dux, L., and Keller-Pintér, A. (2019). Changes in Redox Signaling in the Skeletal Muscle with Aging. Oxid. Med. Cell. Longevity 2019, 1-12. doi: 10.1155/2019/4617801

Vader, P., Mol, E. A., Pasterkamp, G., and Schiffelers, R. M. (2016). Extracellular vesicles for drug delivery. Adv. Drug Deliv. Rev. 106, 148-156. doi: 10.1016/ j.addr.2016.02.006

Verhaart, I., and Aartsma-Rus, A. (2019). Therapeutic developments for Duchenne muscular dystrophy. Nat. Rev. Neurol. 15 (7), 373-386. doi: 10.1038/s41582-019-0203-3
Waning, D. L., Mohammad, K. S., Reiken, S., Xie, W., Andersson, D. C., John, S., et al. (2015). Excess TGF-beta mediates muscle weakness associated with bone metastases in mice. Nat. Med. 21 (11), 1262-1271. doi: 10.1038/nm.3961

Weyh, C., Kruger, K., and Strasser, B. (2020). Physical Activity and Diet Shape the Immune System during Aging. Nutrients 12 (3), 622. doi: 10.3390/nu12030622

White, T. A., and LeBrasseur, N. K. (2014). Myostatin and sarcopenia: opportunities and challenges - a mini-review. Gerontology 60 (4), 289-293. doi: 10.1159/000356740

Whitham, M., Parker, B. L., Friedrichsen, M., Hingst, J. R., Hjorth, M., Hughes, W. E., et al. (2018). Extracellular Vesicles Provide a Means for Tissue Crosstalk during Exercise. Cell Metab. 27 (1), 237-251. doi: 10.1016/j.cmet.2017.12.001

Wilkinson, D. J., Piasecki, M., and Atherton, P. J. (2018). The age-related loss of skeletal muscle mass and function: Measurement and physiology of muscle fibre atrophy and muscle fibre loss in humans. Ageing Res. Rev. 47, 123-132. doi: 10.1016/j.arr.2018.07.005

Wilson, D., Jackson, T., Sapey, E., and Lord, J. M. (2017). Frailty and sarcopenia: The potential role of an aged immune system. Ageing Res. Rev. 36, 1-10. doi: 10.1016/j.arr.2017.01.006

Woodhouse, L., Gandhi, R., Warden, S. J., Poiraudeau, S., Myers, S. L., Benson, C. T., et al. (2016). A Phase 2 Randomized Study Investigating the Efficacy and Safety of Myostatin Antibody LY2495655 versus Placebo in Patients Undergoing Elective Total Hip Arthroplasty. J. Frailty Aging 5 (1), 62-70. doi: $10.14283 /$ jfa.2016.81

Yin, H., Price, F., and Rudnicki, M. A. (2013). Satellite Cells and the Muscle Stem Cell Niche. Physiol. Rev. 93 (1), 23-67. doi: 10.1152/physrev.00043.2011

Yin, P., Li, Y., Lv, H., Deng, Y., Meng, Y., Zhang, L., et al. (2018). Exchange of genetic material: a new paradigm in bone cell communications. Cell. Mol. Life Sci. 75 (11), 1989-1998. doi: 10.1007/s00018-018-2782-3

Zammit, P. S. (2017). Function of the myogenic regulatory factors Myf5, MyoD, Myogenin and MRF4 in skeletal muscle, satellite cells and regenerative myogenesis. Semin. Cell Dev. Biol. 72, 19-32. doi: 10.1016/j.semcdb.2017.11.011

Zanotti, S., Gibertini, S., Blasevich, F., Bragato, C., Ruggieri, A., Saredi, S., et al. (2018). Exosomes and exosomal miRNAs from muscle-derived fibroblasts promote skeletal muscle fibrosis. Matrix Biol. 74, 77-100. doi: 10.1016/ j.matbio.2018.07.003

Zhou, T., Prather, E. R., Garrison, D. E., and Zuo, L. (2018). Interplay between ROS and Antioxidants during Ischemia-Reperfusion Injuries in Cardiac and Skeletal Muscle. Int. J. Mol. Sci. 19 (2), 417. doi: 10.3390/ijms19020417

Zhu, P., Zhang, C., Gao, Y., Wu, F., Zhou, Y., and Wu, W. (2019). The transcription factor Slug represses p16Ink4a and regulates murine muscle stem cell aging. Nat. Commun. 10 (1), 2568. doi: 10.1038/s41467-019-10479-4

Conflict of Interest: The authors declare that the research was conducted in the absence of any commercial or financial relationships that could be construed as a potential conflict of interest.

Copyright (c) $2020 \mathrm{Li}$, Chen, Zhao, Li, Qin, Cheng, Yang, Yin, Zhang and Tang. This is an open-access article distributed under the terms of the Creative Commons Attribution License (CC BY). The use, distribution or reproduction in other forums is permitted, provided the original author(s) and the copyright owner(s) are credited and that the original publication in this journal is cited, in accordance with accepted academic practice. No use, distribution or reproduction is permitted which does not comply with these terms. 